$\mathrm{J}$ o u r n a l of

Mathematics

and Applications

JMA No 40, pp 135-148 (2017)

\title{
Approximation by Szász Type Operators Including Sheffer Polynomials
}

\author{
Nadeem Rao, Abdul Wafi and Deepmala
}

\begin{abstract}
In present article, we discuss voronowskaya type theorem, weighted approximation in terms of weighted modulus of continuity for Szász type operators using Sheffer polynomials. Lastly, we investigate statistical approximation for these sequences.
\end{abstract}

AMS Subject Classification: 41A10, 41A25, 41A36.

Keywords and Phrases: Szász operators; Sheffer Polynomials; Voronovskaya.

\section{Introduction}

First, we recall $n^{\text {th }}$ Bernstein operators due to Bernstein [1] defined as follows

$$
B_{n}(f ; x)=\sum_{k=0}^{n} p_{n, k}(x) f\left(\frac{k}{n}\right)
$$

where $p_{n, k}(x)=\left(\begin{array}{l}n \\ k\end{array}\right) x^{k}(1-x)^{n-k}, f \in C[0,1]$ and $0 \leq x \leq 1$. The purpose of this probabilistic method was to prove Weierstass approximation theorem more elegantly. In 1950, Szász [6] generalized operators given by (1.1) for unbounded interval on the space of continuous functions defined on $(0, \infty)$ as

$$
S_{n}(f ; x)=e^{-n x} \sum_{k=0}^{\infty} \frac{(n x)^{k}}{k !} f\left(\frac{k}{n}\right), \quad \forall x \in(0, \infty), \quad n \in \mathbb{N} \text {. }
$$

A new type of generalization of Szász-Mirakjan operators which involves Appell polynomials was given by Jakimovski and Leviatan [4] as follows

$$
P_{n}(f ; x)=\frac{e^{-n x}}{A(1)} \sum_{k=0}^{\infty} p_{k}(n x) f\left(\frac{k}{n}\right) .
$$

COPYRIGHT (C) by Publishing House of Rzeszów University of Technology P.O. Box 85, 35-959 Rzeszów, Poland 
In above relation $p_{k}$ are Appell polynomials defined by the generating functions

$$
A(u) e^{u x}=\sum_{k=0}^{\infty} p_{k}(x) u^{k},
$$

where $A(z)=\sum_{k=0}^{\infty} a_{k} z^{k}\left(a_{0}=0\right)$ is an analytic function in the disc $|z|<R(R>1)$ and $A(z) \neq 0$. A more generalized form of Szász operators including Sheffer polynomials was given by Ismail [3]

$$
T_{n}(f ; x)=\frac{e^{-n x H(1)}}{A(1)} \sum_{k=0}^{\infty} p_{k}(n x) f\left(\frac{k}{n}\right) .
$$

In above relation $p_{k}$ are Sheffer polynomials given by the generating functions

$$
A(u) e^{x H(u)}=\sum_{k=0}^{\infty} p_{k}(x) u^{k}
$$

where

$$
\begin{array}{ll}
A(z)=\sum_{k=0}^{\infty} a_{k} z^{k} \quad & \left(a_{0} \neq 0\right) \\
H(z)=\sum_{k=0}^{\infty} h_{k} z^{k} & \left(h_{1} \neq 0\right)
\end{array}
$$

be analytic functions in the disc $|z|<R(R>1)$. Under the following restrictions:

(i) for $x \in[0, \infty)$ and $k \in N \cup 0, p_{k}(x) \geq 0$,

(ii) $A(1) \neq 0$ and $H^{\prime}(1)=1$,

(iii) relation (1.4) is valid for $|u|<R$ and the power series given by (1.5) converges for $|z|<R, R>1$. Moreover, Ismail introduced the Kantorovich form of the operator (1.3) as

$$
T_{n}^{*}(f ; x)=n \frac{e^{-n x H(1)}}{A(1)} \sum_{k=0}^{\infty} p_{k}(n x) \int_{\frac{k}{n}}^{\frac{k+1}{n}} f(s) d s .
$$

Recently, Sucu and Ertan Ibikli [7] proved results on rate of convergence using modulus of continuity for (1.3) and (1.6). Motivated by the above development, we prove weighted approximation, statistical approximation and Voronovskaya type result for $T_{n}$ in the present paper.

Various investigators such as Gairola et al. [9], Singh et al. [10], Mishra et al. [1621], Gandhi et al. [22] and the references therein, have discussed the approximation properties of various linear positive operators in this direction. 


\section{Some properties of the operator $T_{n}$}

We recall following lemmas due to Sezgin et al. [7]:

Lemma 2.1. Let $e_{i}=t^{i}, i=0,1,2, x \in[0, \infty)$, we have

$$
\begin{aligned}
& T_{n}\left(e_{0} ; x\right)=1, \\
& T_{n}\left(e_{1} ; x\right)=x+\frac{A^{\prime}(1)}{n A(1)} \\
& T_{n}\left(e_{2} ; x\right)=x^{2}+\left(\frac{2 A^{\prime}(1)}{A(1)}+H^{\prime \prime}(1)+1\right) \frac{x}{n}+\frac{A^{\prime}(1)+A^{\prime \prime}(1)}{n^{2} A(1)} .
\end{aligned}
$$

Lemma 2.2. Let $\psi_{x}^{i}(t)=(t-x)^{i}, i=0,1,2$, for $x \geq 0$ and $n \in N$ we have

$$
\begin{aligned}
T_{n}\left(\psi_{x}^{0}(t) ; x\right) & =1 \\
T_{n}\left(\psi_{x}^{1}(t) ; x\right) & =\frac{A^{\prime}(1)}{n A(1)} \\
T_{n}\left(\psi_{x}^{2}(t) ; x\right) & =\left(\frac{H^{\prime \prime}(1)+1}{n}\right)+\frac{A^{\prime}(1)+A^{\prime \prime}(1)}{n^{2} A(1)} .
\end{aligned}
$$

Next we prove

Lemma 2.3. For $x \geq 0$, we have

$$
\begin{aligned}
T_{n}\left(e_{3} ; x\right)= & x^{3}+\left(3+\frac{3 A^{\prime}(1)}{A(1)}+3 H^{\prime \prime}(1)\right) \frac{x^{2}}{n} \\
& +\left(\frac{2+3 A^{\prime \prime}(1)}{A(1)}+\frac{6 A^{\prime}(1)}{A(1)}+\frac{3 A^{\prime}(1) H^{\prime \prime}(1)}{A(1)}+H^{\prime \prime}(1)+H^{\prime \prime \prime}(1)\right) \frac{x}{n^{2}} \\
& +\frac{2 A^{\prime}(1)+3 A^{\prime \prime}(1)+A^{\prime \prime \prime}(1)}{n^{3} A(1)}, \\
T_{n}\left(e_{4} ; x\right)= & x^{4}+\left(6+\frac{4 A^{\prime}(1)}{A(1)}+6 H^{\prime \prime}(1)\right) \frac{x^{3}}{n} \\
& +\left(11+\frac{6 A^{\prime \prime}(1)}{A(1)}+\frac{18 A^{\prime}(1)}{A(1)}+18 H^{\prime \prime}(1)+\frac{9 A^{\prime}(1) H^{\prime \prime}(1)}{A(1)}+3\left(H^{\prime \prime}(1)\right)^{2}\right. \\
& \left.+4 H^{\prime \prime \prime}(1)\right) \frac{x^{2}}{n^{2}}+\left(6+\frac{4 A^{\prime \prime \prime}(1)}{A(1)}+\frac{18 A^{\prime \prime}(1)}{A(1)}+\frac{22 A^{\prime}(1)}{A(1)}+\frac{6 A^{\prime \prime}(1) H^{\prime \prime}(1)}{A(1)}\right. \\
& \left.+\frac{18 A^{\prime}(1) H^{\prime \prime}(1)}{A(1)}+\frac{4 A^{\prime}(1) H^{\prime \prime \prime}(1)}{A(1)}+6 H^{\prime \prime \prime}(1)+11 H^{\prime \prime}(1)+H^{\prime \prime \prime \prime}(1)\right) \frac{x}{n^{3}} \\
& +\frac{6 A^{\prime}(1)+11 A^{\prime \prime}(1)+A^{\prime \prime \prime \prime}(1)}{A(1)} .
\end{aligned}
$$


Proof. From the generating functions of Sheffer polynomials, we obtain

$$
\begin{aligned}
\sum_{K=0}^{\infty} K^{3} P_{K}(n x)= & {\left[\left(2 A^{\prime}(1)+3 A^{\prime \prime}(1)+A^{\prime \prime \prime}(1)\right)\right.} \\
& +n x\left(3 A^{\prime \prime}(1)+6 A^{\prime}(1)+3 A^{\prime}(1) H^{\prime \prime}(1)\right. \\
& \left.+3 A(1) H^{\prime \prime}(1)+2 A(1)+A(1) H^{\prime \prime \prime}(1)\right)+n^{2} 2 x^{2}\left(3 A(1)+3 A^{\prime}(1)\right. \\
& \left.\left.+3 A(1) H^{\prime \prime}(1)\right)+n^{3} x^{3} A(1)\right] e^{n} x H(1), \\
\sum_{K=0}^{\infty} K^{4} P_{K}(n x)= & {\left[\left(6 A^{\prime}(1)+11 A^{\prime \prime}(1)+6 A^{\prime \prime \prime}(1)\right.\right.} \\
& \left.+A^{\prime \prime \prime \prime}(1)\right)+n x\left(4 A^{\prime \prime \prime}(1)+18 A^{\prime \prime}(1)\right. \\
& +22 A^{\prime}(1)+6 A^{\prime \prime}(1) H^{\prime \prime}(1)+18 A^{\prime}(1) H^{\prime \prime}(1)+4 A^{\prime}(1) H^{\prime \prime \prime}(1) \\
& \left.+6 A(1) H^{\prime \prime \prime}(1)+1 A^{\prime \prime}(1) H^{\prime \prime}(1)+6 A(1)+A(1) H^{\prime \prime \prime \prime}\right) \\
& +n^{2} x^{2}\left(11 A(1)+18 A^{\prime}(1)\right. \\
& +8 A(1) H^{\prime \prime}(1)+6 A^{\prime \prime}(1)+9 A^{\prime}(1) H^{\prime \prime}(1) \\
& \left.+3 A(1)\left(H^{\prime \prime}(1)\right)^{2}+4 A(1) H^{\prime \prime \prime}(1)\right)+n^{3} x^{3}\left(6 A(1)+4 A^{\prime}(1)\right. \\
& \left.\left.+6 A(1) H^{\prime \prime}(1)\right)+n^{4} x^{4} A(1)\right] e^{n} x H(1) . \\
&
\end{aligned}
$$

The proof of Lemma 2.3 is obvious using these relation.

Lemma 2.4. The operator (1.3) satisfies the following relation:

$$
\begin{aligned}
T_{n}\left(\psi_{x}^{4}(t) ; x\right)= & \left(3+14 H^{\prime \prime}(1)+\frac{3 A^{\prime}(1) H^{\prime \prime}(1)}{A(1)}+3\left(H^{\prime \prime}\right)^{2}+4 H^{\prime \prime}(1)\right) \frac{x^{2}}{n^{2}} \\
& +\left(6+\frac{6 A^{\prime \prime}(1)}{A(1)}+\frac{14 A^{\prime}(1)}{A(1)}+\frac{6 A^{\prime \prime}(1) H^{\prime \prime}(1)}{A(1)}+\frac{18 A^{\prime}(1) H^{\prime \prime}(1)}{A(1)}\right. \\
& +\frac{4 A^{\prime \prime}(1) H^{\prime \prime}(1)}{A(1)}+\frac{6 A^{\prime \prime}(1) H^{\prime \prime}(1)}{A(1)} \\
& \left.+6 H^{\prime \prime \prime}(1)+11 H^{\prime \prime}(1)+H^{\prime \prime \prime \prime}(1)\right) \frac{x}{n^{3}} \\
& +\frac{6 A^{\prime}(1)+11 A^{\prime \prime}(1)+A^{\prime \prime \prime \prime}(1)}{n^{4} A(1)} .
\end{aligned}
$$

Proof. Proof of this relation can be obtained using Lemma 2.1 and linearity property 
of the operators

$T_{n}\left((t-x)^{4} ; x\right)=T_{n}\left(t^{4} ; x\right)-4 x T_{n}\left(t^{3} ; x\right)+6 x^{2} T_{n}\left(t^{2} ; x\right)-4 x^{3} T_{n}(t ; x)+T_{n}(1 ; x)$.

\section{The Voronovskaya type theorem for $T_{n}$}

Theorem 3.1. Let $f \in C^{2}[0, b]$. Then $\forall x \in[0, b]$, we have

$$
\lim _{n \rightarrow \infty} n\left\{T_{n}(f ; x)-f(x)\right\}=\frac{A^{\prime}(1)}{A(1)} f^{\prime}(x)+\left(H^{\prime \prime}(1)+1\right) x \frac{f^{\prime \prime}(x)}{2 !} .
$$

Proof. Let $x_{0} \in[0, b]$ be a fixed point. Then for $f \in C^{2}[0, b]$ and $t \in[0, b]$ we have by Taylor's formula

$$
f(t)=f\left(x_{0}\right)+f^{\prime}\left(x_{0}\right)\left(t-x_{0}\right)+\frac{1}{2} f^{\prime \prime}\left(x_{0}\right)(t-x)^{2}+\varphi\left(t ; x_{0}\right)\left(t-x_{0}\right)^{2},
$$

where $\varphi\left(t ; x_{0}\right) \in C[0, b]$ and $\lim _{t \rightarrow x_{0}} g\left(t ; x_{0}\right)=0$. Now, applying the operator on both the side and in the light of linearity property, we have

$$
\begin{aligned}
T_{n}(f ; x)= & f\left(x_{0}\right) T_{n}\left(1 ; x_{0}\right)+f^{\prime}\left(x_{0}\right) T_{n}\left(\left(t-x_{0}\right) ; x_{0}\right)+\frac{1}{2} f^{\prime \prime}\left(x_{0}\right) T_{n}\left((t-x)^{2} ; x_{0}\right) \\
& \left.+T_{n}\left(\varphi\left(t ; x_{0}\right)\left(t-x_{0}\right)^{2} ; x_{0}\right)\right) .
\end{aligned}
$$

Subtract $f\left(x_{0}\right)$ and then on multiplying by $n$ both side, we obtain

$$
\begin{aligned}
n\left\{T_{n}\left(f ; x_{0}\right)-f\left(x_{0}\right)\right\}= & f^{\prime}\left(x_{0}\right) n T_{n}\left(\left(t-x_{0}\right) ; x_{0}\right)+\frac{f^{\prime \prime}\left(x_{0}\right)}{2} n T_{n}\left(\left(t-x_{0}\right)^{2} ; x_{0}\right) \\
& +n T_{n}\left(\varphi\left(t ; x_{0}\right)(t-x)^{2} ; x_{0}\right) .
\end{aligned}
$$

We have

$$
\begin{aligned}
\lim _{n \rightarrow \infty} n\left\{T_{n}(f ; x)-f(x)\right\}= & \frac{A^{\prime}(1)}{A(1)} f^{\prime}(x)+\left(H^{\prime \prime}(1)+1\right) x \frac{f^{\prime \prime}(x)}{2 !} \\
& +\lim _{n \rightarrow \infty} n T_{n}\left(\varphi\left(t ; x_{0}\right)(t-x)^{2} ; x_{0}\right)
\end{aligned}
$$

Using Holder's inequality. The last term can be given by

$$
n T_{n}\left(\varphi\left(t ; x_{0}\right)(t-x)^{2} ; x_{0}\right) \leq n^{2} T_{n}\left((t-x)^{4} ; x_{0}\right) T_{n}\left(\varphi\left(t ; x_{0}\right)^{2} ; x_{0}\right)
$$


Let $\eta\left(t ; x_{0}\right)=\varphi^{2}\left(t ; x_{0}\right)$. Then $\lim \eta\left(t ; x_{0}\right)=\lim \varphi^{2}\left(t ; x_{0}\right)=0$ as $n \rightarrow \infty$. By using

$\lim _{n \rightarrow \infty} n^{2} T_{n}\left(\psi_{x}^{4}(t) ; x\right)=\left(3+14 H^{\prime \prime}(1)+\frac{3 A^{\prime}(1) H^{\prime \prime}(1)}{A(1)}+3\left(H^{\prime \prime}(1)^{2}\right)+4 H^{\prime \prime}(1)\right) x^{2}$,

we get

$$
\lim _{n \rightarrow \infty} n T_{n}\left(\varphi\left(t ; x_{0}\right)(t-x)^{2} ; x_{0}\right)=0
$$

which proves the Theorem 3.1.

\section{Weighted approximation}

Here, we recall some notation from [11] to prove next result. Let $B_{1+x^{2}}[0, \infty)=$ $\left\{f(x):|f(x)| \leq M_{f}\left(1+x^{2}\right), 1+x^{2}\right.$ is weight function, $M_{f}$ is a constant depending on $f$ and $x \in[0, \infty)\}, C_{1+x^{2}}[0, \infty)$ is the space of continuous function in $B_{1+x^{2}}[0, \infty)$ with the norm $\|f(x)\|_{1+x^{2}}=\sup _{x \in[0, \infty)} \frac{|f(x)|}{1+x^{2}}$ and $C_{1+x^{2}}^{k}[0, \infty)=\left\{f \in C_{1+x^{2}}: \lim _{|x| \rightarrow \infty} \frac{f(x)}{1+x^{2}}=\right.$ $k$, where $k$ is a constant depending on $f$ \}.

Modulus of continuity for the function $f$ defined on closed interval $[0, a]$ with $a>0$ is denoted as follows

$$
\omega_{a}(f, \delta)=\sup _{|t-x| \leq \delta} \sup _{x, t \in[0, a]}|f(t)-f(x)| .
$$

Theorem 4.1. Let $f \in C_{1+x^{2}}[0, \infty)$ and $\omega_{b+1}(f ; \delta)$ be its modulus of continuity defined on $[0, b+1] \subset[0, \infty)$. Then, we have

$$
\left\|T_{n}(f ; x)-f(x)\right\|_{C[0, b]} \leq 6 M_{f}\left(1+b^{2}\right) \delta_{n}(b)+2 \omega_{b+1}\left(f ; \sqrt{\delta_{n}(b)}\right),
$$

where $\delta_{n}(b)=T_{n}\left(\psi_{b}^{2} ; b\right)$.

Proof. From ([12], p. 378), for $x \in[0, b]$ and $t \in[0, \infty)$, we have

$$
|f(t)-f(x)| \leq 6 M_{f}\left(1+b^{2}\right)(t-x)^{2}+\left(1+\frac{|t-x|}{\delta}\right) \omega_{b+1}(f ; \delta) .
$$

This implies that

$\left|T_{n}(f ; x)-f(x)\right| \leq 6 M_{f}\left(1+b^{2}\right) T_{n}\left((t-x)^{2} ; x\right)+\left(1+\frac{T_{n}(|t-x| ; x)}{\delta}\right) \omega_{b+1}(f ; \delta)$.

Thus, using Lemma 2.4, for $x \in[0, b]$, we have 


$$
\left|T_{n}(f ; x)-f(x)\right| \leq 6 M_{f}\left(1+b^{2}\right) \delta_{n}(b)+\left(1+\frac{\sqrt{\delta_{n}(b)}}{\delta}\right) \omega_{b+1}(f ; \delta) .
$$

Choosing $\delta=\delta_{n}(b)$, we arrive at the desired result.

Theorem 4.2. If the operators $T_{n}$ defined by (1.3) from $C_{1+x^{2}}^{k}[0, \infty)$ to $B_{1+x^{2}}[0, \infty)$ satisfying the conditions

$$
\lim _{n \rightarrow \infty}\left\|T_{n}\left(e_{i} ; x\right)-x^{i}\right\|_{1+x^{2}}=0, \quad i=0,1,2,
$$

then for each $C_{1+x^{2}}^{k}[0, \infty)$

$$
\lim _{n \rightarrow \infty}\left\|T_{n}(f ; x)-f\right\|_{1+x^{2}}=0 .
$$

Proof. To prove this Theorem, it is enough to show that

$$
\lim _{n \rightarrow \infty}\left\|T_{n}\left(e_{i} ; x\right)-x^{i}\right\|_{1+x^{2}}=0, \quad i=0,1,2 .
$$

From Lemma 2.2, we have

$$
\left\|T_{n}\left(e_{0} ; x\right)-x^{0}\right\|_{1+x^{2}}=\sup _{x \in[0, \infty)} \frac{\left|T_{n}(1 ; x)-1\right|}{1+x^{2}}=0 \text { for } i=0 .
$$

For $i=1$

$$
\begin{aligned}
\left\|T_{n}\left(e_{1} ; x\right)-x^{1}\right\|_{1+x^{2}} & =\sup _{x \in[0, \infty)} \frac{\frac{A^{\prime}(1)}{n A(1)}}{1+x^{2}} \\
& =\frac{A^{\prime}(1)}{n A(1)} \sup _{x \in[0, \infty)} \frac{1}{1+x^{2}} .
\end{aligned}
$$

This implies that $\left\|T_{n}\left(e_{1} ; x\right)-x^{1}\right\|_{1+x^{2}} \rightarrow 0$ an $n \rightarrow \infty$. For $i=2$

$$
\begin{aligned}
\left\|T_{n}\left(e_{2} ; x\right)-x^{2}\right\|_{1+x^{2}} & =\sup _{x \in[0, \infty)} \frac{\left|\left(\frac{2 A^{\prime}(1)}{A(1)}+H^{\prime \prime}(1)+1\right) \frac{x}{n}+\frac{A^{\prime}(1)+A^{\prime \prime}(1)}{n^{2} A(1)}\right|}{1+x^{2}} \\
& \leq \frac{\left(\frac{2 A^{\prime}(1)}{A(1)}+H^{\prime \prime}(1)+1\right)}{n} \sup _{x \in[0, \infty)} \frac{x}{1+x^{2}} \\
& +\frac{A^{\prime}(1)+A^{\prime \prime}(1)}{n^{2} A(1)} \sup _{x \in[0, \infty)} \frac{1}{1+x^{2}} .
\end{aligned}
$$

Which shows that $\left\|T_{n}\left(e_{2} ; x\right)-x^{2}\right\|_{1+x^{2}} \rightarrow 0$ an $n \rightarrow \infty$. 
Let $f \in C_{\rho}^{k}[0, \infty)$, Yüksel and Ispir [13] introduced weighted modulus of continuity as follows

$$
\Omega(f ; \delta)=\sup _{x \in[0, \infty), 0<h \leq \delta} \frac{|f(x+h)-f(x)|}{1+(x+h)^{2}} .
$$

Theorem 4.3. Let $f \in C_{1+x^{2}}^{k}[0, \infty)$. Then

(i) $\Omega(f ; \delta)$ is a monotone increasing function of $\delta$;

(ii) $\lim _{\delta \rightarrow 0^{+}} \Omega(f ; \delta)=0$;

(iii) for each $m \in \mathbb{N}, \Omega(f ; m \delta) \leq m \Omega(f ; \delta)$;

(iv) for each $\lambda \in[0, \infty), \Omega(f ; \lambda \delta) \leq(1+\lambda) \Omega(f ; \delta)$

and for $t, x \in[0, \infty)$, one get

$$
|f(t)-f(x)| \leq 2\left(1+\frac{|t-x|}{\delta}\right)\left(1+\delta^{2}\right)\left(1+x^{2}\right)\left(1+(t-x)^{2}\right) \Omega(f ; \delta) .
$$

Theorem 4.4. Let $f \in C_{1+x^{2}}^{k}[0, \infty)$. Then, we have

$$
\sup _{x \in[0, \infty)} \frac{\left|T_{n}(f ; x)-f(x)\right|}{\left(1+x^{2}\right)^{3}} \leq C\left(1+\frac{1}{n}\right) \Omega\left(f ; \frac{1}{\sqrt{n}}\right),
$$

where $C>0$ is a constant.

Proof. Using (4.2) and $x, t \in(0, \infty)$, we have

$$
\begin{aligned}
& \left|T_{n}(f ; x)-f(x)\right| \leq 2\left(1+\frac{T_{n}(|t-x| ; x)}{\delta}\right)\left(1+\delta^{2}\right)\left(1+x^{2}\right) \\
& \times\left(1+T_{n}\left((t-x)^{2} ; x\right)\right) \Omega(f ; \delta) .
\end{aligned}
$$

Applying Cauchy-Schwarz inequality for (4.2), we get

$$
\begin{aligned}
\left|T_{n}(f ; x)-f(x)\right| & \leq 2\left(1+\delta^{2}\right)\left(1+x^{2}\right) \Omega(f ; \delta)\left(1+T_{n}\left((t-x)^{2} ; x\right)\right. \\
& \left.+\frac{\sqrt{T_{n}\left((t-x)^{2} ; x\right)}}{\delta}+\frac{\sqrt{T_{n}\left((t-x)^{2} ; x\right) T_{n}\left((t-x)^{4} ; x\right)}}{\delta}\right) .
\end{aligned}
$$

Using Lemma 2.2 and Lemma 2.4, we get 


$$
T_{n}\left((t-x)^{2} ; x\right) \leq C_{1} \frac{(1+x)}{n} \text { and } T_{n}\left((t-x)^{4} ; x\right) \leq C_{2} \frac{\left(1+x+x^{2}+x^{3}\right)}{n} .
$$

From and (4.3), we have

$$
\begin{aligned}
\left|T_{n}(f ; x)-f(x)\right| & \leq 2\left(1+\delta^{2}\right)\left(1+x^{2}\right) \Omega(f ; \delta)\left(1+C_{1} \frac{(1+x)}{n}\right. \\
& \left.+\frac{\sqrt{C_{1} \frac{(1+x)}{n}}}{\delta}+\frac{\sqrt{C_{1} \frac{(1+x)}{n} C_{2} \frac{\left(1+x+x^{2}+x^{3}\right)}{n}}}{\delta}\right)
\end{aligned}
$$

On choosing $\delta=\frac{1}{\sqrt{n}}$ and $C=\left\{1+C_{1}+\sqrt{C_{1}}+\sqrt{C_{1} C_{2}}\right\}$, we get the required result.

Theorem 4.5. For $f \in C_{1+x^{2}}^{k}[0, \infty)$ and $\theta>0$, we have

$$
\lim _{n \rightarrow \infty} \sup _{x \in[0, \infty)} \frac{\left|T_{n}(f ; x)\right|-f(x)}{\left(1+x^{2}\right)^{1+\theta}}=0 .
$$

Proof. For any fixed real number $x_{0}>0$, one has say

$$
\begin{aligned}
\sup _{x \in[0, \infty)} \frac{\left|T_{n}(f ; x)\right|-f(x)}{\left(1+x^{2}\right)^{1+\theta}} & \leq \sup _{x \leq x_{0}} \frac{\left|T_{n}(f ; x)\right|-f(x)}{\left(1+x^{2}\right)^{1+\theta}}+\sup _{x \geq x_{0}} \frac{\left|T_{n}(f ; x)\right|-f(x)}{\left(1+x^{2}\right)^{1+\theta}} \\
& \leq\left\|T_{n}(f ; x) \mid-f(x)\right\|_{C\left[0, x_{0}\right]} \\
& +\|f\|_{1+x^{2}} \sup _{x \geq x_{0}} \frac{\left|T_{n}\left(1+t^{2} ; x\right)\right|}{\left(1+x^{2}\right)^{1+\theta}}+\sup _{x \geq x_{0}} \frac{|f(x)|}{\left(1+x^{2}\right)^{1+\theta}} \\
& =I_{1}+I_{2}+I_{3} .
\end{aligned}
$$

Since $|f(x)| \leq\|f\|_{1+x^{2}}\left(1+x^{2}\right)$, we have

$$
\begin{aligned}
I_{3} & =\sup _{x \geq x_{0}} \frac{|f(x)|}{\left(1+x^{2}\right)^{1+\theta}} \\
& \leq \sup _{x \geq x_{0}} \frac{\|f\|_{1+x^{2}}\left(1+x^{2}\right)}{\left(1+x^{2}\right)^{1+\theta}} \leq \frac{\|f\|_{1+x^{2}}}{\left(1+x^{2}\right)^{\theta}} .
\end{aligned}
$$

Let $\epsilon>0$ be arbitrary real number. Then, from Theorem 4.2 there exists $n_{1} \in \mathbb{N}$ such that

$$
\begin{aligned}
I_{2} & <\frac{1}{\left(1+x^{2}\right)^{\theta}}\|f\|_{1+x^{2}}\left(1+x^{2}+\frac{\epsilon}{3\|f\|_{1+x^{2}}}\right) \text { for all } n_{1} \geq n \\
& <\frac{\|f\|_{1+x^{2}}}{\left(1+x^{2}\right)^{\theta}}+\frac{\epsilon}{3} \text { for all } n_{1} \geq n .
\end{aligned}
$$

This implies that

$$
I_{2}+I_{3}<2 \frac{\|f\|_{1+x^{2}}}{\left(1+x^{2}\right)^{\theta}}+\frac{\epsilon}{3}
$$


Next, let for a large value of $x_{0}$, we have $\frac{\|f\|_{1+x^{2}}}{\left(1+x^{2}\right)^{\theta}}<\frac{\epsilon}{6}$.

$$
I_{2}+I_{3}<\frac{2 \epsilon}{3} \text { for all } n_{1} \geq n .
$$

From Theorem 4.2, there exists $n_{2}>n$ in such a way

$$
I_{1}=\left\|T_{n}(f)-f\right\|_{C\left[0, x_{0}\right]}<\frac{\epsilon}{3} \text { for all } n_{2} \geq n .
$$

Let $n_{3}=\max \left(n_{1}, n_{2}\right)$. Then, combining (4.6), (4.7) and (4.8), we have

$$
\sup _{x \in[0, \infty)} \frac{\left|T_{n}(f ; x)\right|-f(x)}{\left(1+x^{2}\right)^{1+\theta}}<\epsilon .
$$

Hence, the proof of Theorem 4.5 is completed.

\section{A-statistical approximation}

Gadjiev et al. [14] was the first who introduced Statistical approximation theorems in operators theory. Here, we recall same notation from [14], let $A=\left(a_{n k}\right)$ be a nonnegative infinite suitability matrix. For a given sequence $x:=\left(x_{k}\right)$, the $A$-transform of $x$ denoted by $A x:\left((A x)_{n}\right)$ is defined as

$$
(A x)_{n}=\sum_{k=1}^{\infty} a_{n k} x_{k},
$$

provided the series converges for each $n$. A is said to be regular if $\lim (A x)_{n}=L$ whenever $\lim x=L$. Then $x=\left(x_{n}\right)$ is said to be a $A$-statistically convergent to $L$ i.e. $s t_{A}-\lim x=L$ if for every $\epsilon>0, \lim _{n} \sum_{k:\left|x_{k}-L\right| \geq \epsilon} a_{n k}=0$.

Theorem 5.1. Let $A=\left(a_{n k}\right)$ be a non-negative regular suitability matrix and $x \geq 0$. Then, we have

$$
s t_{A}-\lim _{n}\left\|T_{n}(f ; x)-f\right\|_{1+x^{2+\lambda}}=0 \text {, for all } f \in C_{1+x^{2+\lambda}}^{k}[0, \infty) \text { and } \lambda>0 .
$$

Proof. From ([15], p. 191, Th. 3), it is sufficient to show that for $\lambda=0$

$$
s t_{A}-\lim _{n}\left\|T_{n}\left(e_{i} ; x\right)-e_{i}\right\|_{1+x^{2}}=0, \text { for } i \in\{0,1,2\} .
$$

Using Lemma 2.2, we have

$$
\begin{aligned}
\left\|T_{n}\left(e_{1} ; x\right)-x\right\|_{1+x^{2}} & =\sup _{x \in[0, \infty)} \frac{1}{1+x^{2}}\left|\frac{A^{\prime}(1)}{n A(1)}\right| \\
& =\frac{A^{\prime}(1)}{n A(1)} \sup _{x \in[0, \infty)} \frac{1}{1+x^{2}}
\end{aligned}
$$


Now, for a given $\epsilon>0$, we define the following sets

$$
\begin{aligned}
& M_{1}:=\left\{n:\left\|T_{n}\left(e_{1} ; x\right)-x\right\| \geq \epsilon\right\}, \\
& M_{2}:=\left\{n: \frac{A^{\prime}(1)}{n A(1)} \geq \epsilon\right\} .
\end{aligned}
$$

This implies that $M_{1} \subseteq M_{2}$, which shows that $\sum_{k \in M_{1}} a_{n k} \leq \sum_{k \in M_{2}} a_{n k}$. Hence, we have

$$
s t_{A}-\lim _{n}\left\|T_{n}\left(e_{1} ; x\right)-x\right\|_{1+x^{2}}=0 .
$$

For $i=2$ and using Lemma 2.2, we have

$$
\begin{aligned}
\left\|T_{n}\left(e_{2} ; x\right)-x^{2}\right\|_{1+x^{2}} & =\sup _{x \in[0, \infty)} \frac{x}{1+x^{2}} \mid\left(\frac{2 A^{\prime}(1)}{A(1)}+H^{\prime \prime}(1)+1\right) \frac{1}{n} \\
& +\frac{A^{\prime}(1)+A^{\prime \prime}(1)}{n^{2} A(1)} \sup _{x \in[0, \infty)} \frac{1}{1+x^{2}} .
\end{aligned}
$$

For a given $\varepsilon>0$, we have the following sets

$$
\begin{aligned}
& T_{1}:=\left\{n:\left\|T_{n}\left(e_{2} ; x\right)-x^{2}\right\| \geq \epsilon\right\}, \\
& T_{2}:=\left\{n:\left(\frac{2 A^{\prime}(1)}{A(1)}+H^{\prime \prime}(1)+1\right) \frac{1}{n} \geq \frac{\epsilon}{2}\right\}, \\
& T_{3}:=\left\{n: \frac{A^{\prime}(1)+A^{\prime \prime}(1)}{n^{2} A(1)} \geq \frac{\epsilon}{2}\right\} .
\end{aligned}
$$

This implies that $T_{1} \subseteq T_{2} \bigcup T_{3}$. By which, we get

$$
\sum_{k \in T_{1}} a_{n k} \leq \sum_{k \in T_{2}} a_{n k}+\sum_{k \in T_{3}} a_{n k}
$$

As $n \rightarrow \infty$, we have

$$
s t_{A}-\lim _{n}\left\|T_{n}\left(e_{2} ; x\right)-x^{2}\right\|_{1+x^{2}}=0 .
$$

This completes the proof of Theorem 5.1. 


\section{References}

[1] S.N. Bernstein, Démonstration du théorème de Weierstrass fondée sur le calcul des probabilités, Comm. Soc. Math. Kharkow 213 (1912) 1-2.

[2] P.L. Butzer, R.J. Nessel, Fourier Analysis and Approximation, Birkhäuser, Bessel and Academic Press, New York 11971.

[3] M.E.H. Ismail, On a generalization of Szász operators, Mathematica (Cluj) 39 (1974) 259-267.

[4] A. Jakimovski, D. Leviatan, Generalized Szász operators for the approximation in the infinite interval, Mathematica (Cluj) 11 (1969) 97-103.

[5] L. Rempulska, M. Skorupka, The Voronovskaya theorem for some operators of the Szász-Mirakjan type, Le Matematiche 250 (1995) 251-261.

[6] O. Szász, Generalization of S. Bernstein's polynomials to the infinite interval, J. Research Nat. Bur. Standards 45 (1950) 239-245.

[7] S. Sucu, E. Ibikli, Rate of convergance of Szász type operators including Sheffer polynomials, Stud. Univ. Babes-Bolyai Math. 158 (2013) 55-63.

[8] E. Voronovskaja, Détermination de la forme asymptotique de L'approximation des functions par les polynômes de M. Bernstein, C. R. Acad. Sci. URSS 1932 (1932) 79-85.

[9] A.R. Gairola, Deepmala and L.N. Mishra, Rate of approximation by finite iterates of q-Durrmeyer operators, Proc. Natl. Acad. Sci., India, Sect. A Phys. Sci. 862 (2016) 229-234.

[10] K.K. Singh, A.R. Gairola and Deepmala, Approximation theorems for q-analouge of a linear positive operator by A. Lupas, Int. J. Anal. Appl. 121 (2016) 30-37.

[11] A.D. Gadjiev, Theorems of the type of P.P. Korovkin's theorems, Math. Zametki 205 (1976) 781-786 (in Russian), Math. 20 5-6 (1976) 995-998 (in English).

[12] E. Ibikli, E.A. Gadjieva, The order of approximation of some unbounded functions by the sequence of positive linear operators, Turkish J. Math. 193 (1995) 331-337.

[13] I. Yüksel, N. Ispir, Weighted approximation by a certain family of summation integral-type operators, Comput. Math. Appl. 52 10-11 (2006) 1463-1470.

[14] A.D. Gadjiev, C. Orhan, Some approximation theorems via statistical convergence, Rocky Mountain J. Math. 321 (2007) 129-138.

[15] O. Duman, C. Orhan, Statistical approximation by positive linear operators, Studia Math. 162 (2004) 187-197. 
[16] V.N. Mishra, K. Khatri, L.N. Mishra and Deepmala, Inverse result in simultaneous approximation by Baskakov-Durrmeyer-Stancu operators, Journal of Inequalities and Applications (2013) 2013:586, doi:10.1186/1029-242X-2013-586.

[17] V.N. Mishra, H.H. Khan, K. Khatri and L.N. Mishra, Hypergeometric representation for Baskakov-Durrmeyer-Stancu type operators, Bulletin of Mathematical Analysis and Applications 53 (2013) 18-26.

[18] V.N. Mishra, K. Khatri and L.N. Mishra, On simultaneous approximation for Baskakov-Durrmeyer-Stancu type operators, Journal of Ultra Scientist of Physical Sciences 243 A (2012) 567-577.

[19] V.N. Mishra, K. Khatri and L.N. Mishra, Some approximation properties of $q$ Baskakov-Beta-Stancu type operators, Journal of Calculus of Variations, Volume 2013 (2013) Article ID 814824, 8 pages, http://dx.doi.org/10.1155/2013/814824.

[20] V.N. Mishra, K. Khatri and L.N. Mishra, Statistical approximation by Kantorovich type discrete q-beta operators, Advances in Difference Equations (2013) 2013:345, doi: 10.1186/10.1186/1687-1847-2013-345.

[21] V.N. Mishra, P. Sharma and L.N. Mishra, On statistical approximation properties of q-Baskakov-Szász-Stancu operators, Journal of Egyptian Mathematical Society 243 (2016) 396-401, doi: 10.1016/j.joems.2015.07.005.

[22] R.B. Gandhi, Deepmala and V.N. Mishra, Local and global results for modified Szász-Mirakjan operators, Math. Method. Appl. Sci. (2016), doi: 10.1002/mma.4171.

\title{
DOI: $10.7862 / \mathrm{rf} .2017 .9$
}

\section{Nadeem Rao}

email: nadeemrao1990@gmail.com

Department of Mathematics

Jamia Millia Islamia

New Delhi - 110025

INDIA

\author{
Abdul Wafi \\ email: abdulwafi2k2@gmail.com \\ Department of Mathematics \\ Jamia Millia Islamia \\ New Delhi - 110025 \\ INDIA
}




\section{Deepmala}

email: dmrai23@gmail.com, deepmaladm23@gmail.com

Mathematics Discipline

PDPM Indian Institute of Information Technology

Design and Manufacturing

Jabalpur - 482005

INDIA

Received 30.11.2016

Accepted 18.03.2017 